\title{
The statin era: in search of the ideal lipid regulating agent
}

\author{
J Shepherd
}

Over the past decade, the 3-hydroxy-3methylglutaryl coenzyme A (HMG-CoA) reductase inhibitors, or statins, have established themselves as among the most successful of all classes of cardiovascular drugs. Significant reductions in recurrent myocardial infarction, stroke, and death have been demonstrated in a series of landmark trials of statins in individuals with coronary heart disease (CHD) and high or normal blood cholesterol concentrations. ${ }^{1-3}$ Primary prevention with statins has also been shown in large populations of asymptomatic individuals at increased risk for coronary disease $^{45}$ (fig 1). Aggressive statin treatment has been found to prevent or defer subsequent events in patients after coronary bypass graft surgery ${ }^{6}$ and to be as effective as angioplasty in delaying events in patients with stable angina. ${ }^{?}$ New studies are under way to explore the use of statins to prevent first or recurrent stroke, peripheral vascular disease, diabetic complications, and other conditions.

With their extensive clinical trial track record, statins are by far the most prescribed class of lipid regulating drugs, with worldwide sales that approach US $\$ 10$ billion annually. Although various types of new lipid regulating agents are in development, none appears likely to supplant the statin hegemony in the near future.

Nevertheless, even in the midst of the "statin era" in the management of atherosclerosis, the current crop of HMG-CoA reductase inhibitors leaves ample room for further development and market expansion. Cardiovascular disease remains by far the leading cause of morbidity and mortality in the industrialised world, causing 14.8 million deaths each year. ${ }^{8}$ Despite longstanding evidence that reductions in serum cholesterol concentrations produce reductions in cardiovascular risk, and despite the widespread availability of effective cholesterol lowering treatment, a large majority of patients with CHD or significant risk factors

Institute of

Biochemistry, Glasgow

Royal Infirmary, Glasgow G4 0SF, UK J Shepherd

Correspondence to: Professor Shepherd jshepherd@gri-biochem.org.uk

Accepted 8 November 2000 have serum cholesterol concentrations greater than those recommended by National Cholesterol Education Program (NCEP) or European Joint Task Force guidelines. ${ }^{9} 10$

This article examines the statins as the dominant treatment for the treatment of atherosclerosis in this era, and how they stack up against the hypothetical profile of an ideal lipid regulating agent. In this light, we will

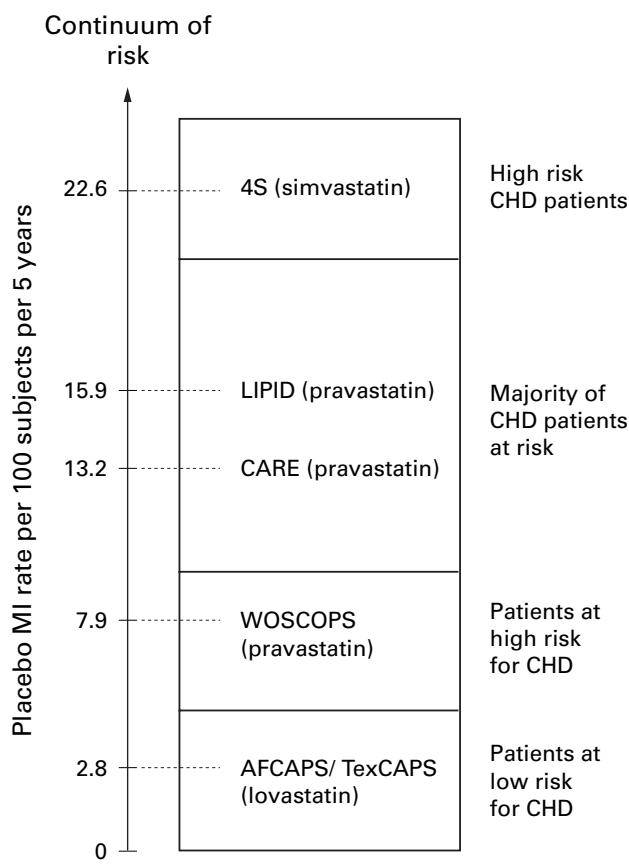

Figure 1 Landmark clinical event trials: relevance to clinical practice. A series of landmark clinical event trials have demonstrated the benefits of statins for primary and secondary prevention. These benefits have been seen in patients with coronary heart disease and high or normal blood cholesterol concentrations, as well as in asymptomatic patients at increased risk for coronary heart disease. $4 S$, Scandinavian simvastatin survival study; LIPID, long-term intervention with pravastatin in ischemic disease; $C A R E$, cholesterol and recurrent events trial; WOSCOPS, West of Scotland coronary prevention study;

AFCAPS/TexCAPS, Air Force/Texas coronary

atherosclerosis prevention study.

briefly review the efficacy and safety records of the available statins, along with a third, broader realm of appropriate utilisation, where today's optimal treatment must continue to advance in order to meet the challenges of 21 st century medicine. Such advances may ultimately come about through combinations of statins with newer developmental agents or, more immediately, through evolution of the statin class itself.

\section{Efficacy of statins}

Statin drugs are structurally homologous to the cholesterol precursor HMG-CoA, which enables them to inhibit HMG-CoA reductase, the enzyme responsible for regulating the rate limiting step in the pathway to cholesterol synthesis. As a result, they limit hepatic cholesterol 
production and upregulate specific high affinity, low density lipoprotein (LDL) receptors located on hepatocyte membranes, thereby limiting the input and promoting the clearance of LDL cholesterol from the circulation.

\section{LDL CHOLESTEROL REDUCTION}

Major epidemiological studies such as the multiple risk factor intervention trial (MRFIT) have shown that the relation between population plasma cholesterol concentrations and coronary risk is curvilinear (fig 2). ${ }^{11}$ On the assumption that cholesterol lowering intervention trials reflect this epidemiological association, it is reasonable to conclude that: (a) the greatest decrements in cardiovascular risk would be gained by treating individuals with higher cholesterol values; and (b) strenuous efforts aimed at driving cholesterol concentrations progressively lower may have limited clinical and economic merit. In that context, post-hoc analyses of the cholesterol and recurrent events (CARE) and long-term intervention with pravastatin in ischemic disease (LIPID) trials suggest that below an LDL cholesterol threshold of $125 \mathrm{mg} / \mathrm{dl}(3.2 \mathrm{mmol} / \mathrm{l})$, further reduction of this lipoprotein fraction does not yield appreciable additional clinical benefit. ${ }^{3}{ }^{12}$ This is consistent with the findings of the West of Scotland coronary prevention study (WOSCOPS), which suggested that there is no linear association between degree of cholesterol reduction and clinical gain. ${ }^{13}$ On the other hand, results from the Scandinavian simvastatin survival study (4S) and the Air Force/Texas coronary atherosclerosis prevention study (AFCAPS/TexCAPS) ${ }^{14}$ appear to support the view that greater LDL reductions yield meas-

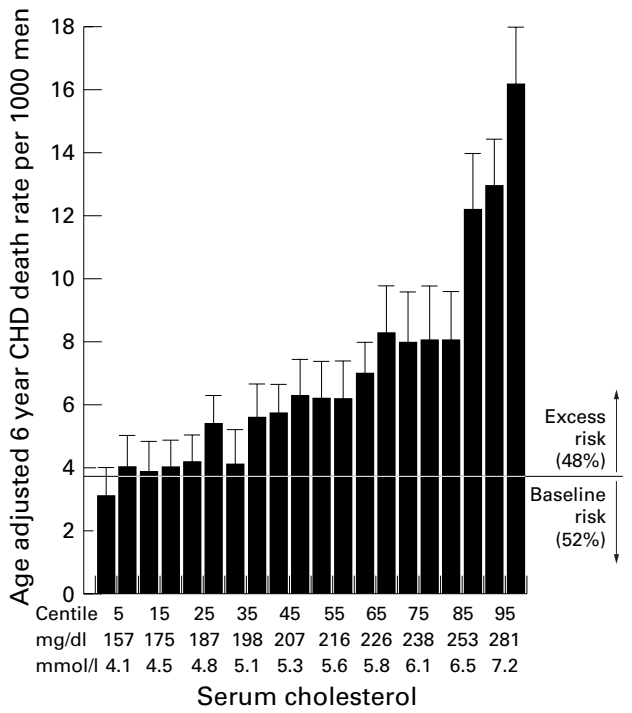

Figure 2 Age adjusted six year CHD death rate per 1000 men screened for the multiple risk factor intervention trial (MRFIT) according to serum cholesterol percentile. The expected death rate of 3.7/1000 is represented by the horizontal line. Area above the line represents excess risk associated with serum cholesterol. $T$ bars represent the upper limit of the $95 \%$ confidence interval. At all points above the 20th centile ( $>181 \mathrm{mg} / \mathrm{dl},>4.68 \mathrm{mmol} / \mathrm{l})$, an increase in serum cholesterol is associated with an increase in coronary heart disease. Reproduced from Martin et al, Lancet 1986;ii:933-6, with permission of the publisher.

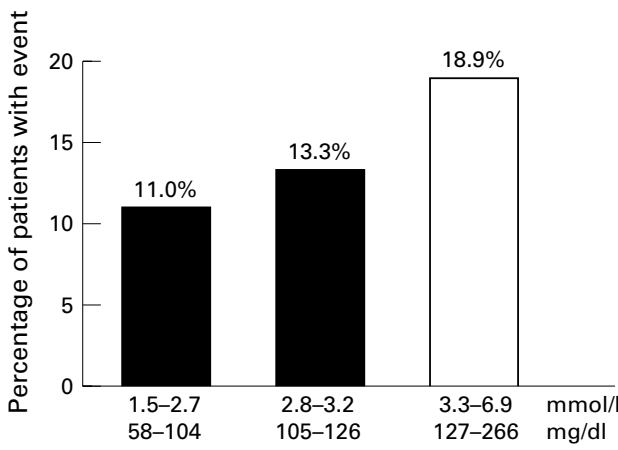

LDL cholesterol after 1 year of treatment (tertiles)

Figure 3 Major coronary events among the simvastatin treated patients in the Scandinavian simvastatin survival study (4S) according to LDL cholesterol concentrations achieved after one year of treatment. $4 S$ supports the view that greater LDL reductions yield measurably improved clinical outcomes: $18.9 \%$ of patients with LDL cholesterol (LDL-C) concentrations between 3.3-6.9 mmol/l

(127-266 mg/dl) had a major coronary event, compared with $11.0 \%$ of patients with LDL-C between 1.5-2.7 $\mathrm{mmol} / \mathrm{l}$ (58-104 mg/dl). Reproduced from Pederson, Eur Heart F 1998;19(suppl M):M15-21, with permission of the publisher.

urably improved clinical outcomes (fig 3), ${ }^{15}$ and the post-coronary artery bypass graft (Post-CABG) trial, though limited in size, produced similar supportive evidence. ${ }^{6}$ Clearly, this issue will not be resolved until the completion of specific studies examining the optimisation of target LDL cholesterol values. Until then, it appears reasonable to endorse either the current US Adult Treatment Panel guidelines calling for an LDL cholesterol reduction to at least $100 \mathrm{mg} / \mathrm{dl}(2.6 \mathrm{mmol} / \mathrm{l})$ or, for Europe, the European Joint Task Force recommended value of $115 \mathrm{mg} / \mathrm{dl}(3.0 \mathrm{mmol} /$ 1) for these higher risk patients. ${ }^{9} 10$

HDL CHOLESTEROL AND TRIGLYCERIDE EFFECTS As noted above, the primary effect of statin drugs is to reduce circulating LDL cholesterol concentrations. The statins also affect high density lipoprotein (HDL) cholesterol and triglyceride parameters, with varying degrees of efficacy. Statins appear to interfere with the synthesis of apolipoprotein B based cholesterol particles, which include triglyceride rich very low density lipoprotein (VLDL), and to promote LDL catabolism. As a result, some statins offer moderately effective triglyceride reduction in patients with hypertriglyceridaemia. Current statins also have a modest HDL cholesterol raising effect of about $6 \%,{ }^{16}$ although some small study evidence suggests that atorvastatin may actually lower HDL cholesterol concentrations at maximum (80 mg) dosage. ${ }^{17}$ Table 1 offers a comparison of current statins in terms of efficacy and pharmacokinetic parameters.

A reasonable case can be made that current statins may be limited in their ability to treat the substantial population of at-risk individuals with lipid abnormalities that are not corrected primarily by LDL cholesterol reduction. Genest and colleagues showed that in patients with documented CHD and lipid disorders, isolated high LDL cholesterol may be less 
Table 1 Characteristics of statins

\begin{tabular}{|c|c|c|c|c|c|c|}
\hline Characteristic & Lovastatin & Pravastatin & Simvastatin & Atorvastatin & Fluvastatin & Cerivastatin \\
\hline Maximal dose (mg/day) & 80 & 40 & 80 & 80 & 40 & 0.4 \\
\hline Maximal serum LDL cholesterol reduction (\%) & 40 & 34 & 47 & 60 & 24 & 28 \\
\hline Serum LDL cholesterol reduction $(\%)^{\star}$ & 34 & 34 & 41 & 50 & 24 & 28 \\
\hline Serum triglyceride reduction $(\%)^{\star}$ & 16 & 24 & 18 & 29 & 10 & 13 \\
\hline Serum HDL cholesterol increase $(\%)^{\star}$ & 8.6 & 12 & 12 & 6 & 8 & 10 \\
\hline Plasma half life (hours) & 2 & $1-2$ & $1-2$ & 14 & 1.2 & $2-3$ \\
\hline Effect of food on drug absorption & Increased absorption & Decreased absorption & None & None & Negligible & None \\
\hline Optimal time of administration & $\begin{array}{l}\text { With meals (morning } \\
\text { and evening) }\end{array}$ & Bedtime & Evening & Evening & Bedtime & Evening \\
\hline Penetration of central nervous system & Yes & No & Yes & No & No & Yes \\
\hline Renal excretion of absorbed dose (\%) & 10 & 20 & 13 & 2 & $<6$ & 33 \\
\hline Mechanism of hepatic metabolism & $\begin{array}{l}\text { Cytochrome P450 } \\
\text { 3A4 }\end{array}$ & Sulfation & $\begin{array}{l}\text { Cytochrome } \\
\text { P450 3A4 }\end{array}$ & $\begin{array}{l}\text { Cytochrome } \\
\text { P450 3A4 }\end{array}$ & $\begin{array}{l}\text { Cytochrome } \\
\text { P450 2C } 9\end{array}$ & $\begin{array}{l}\text { Cytochrome } \\
\text { P450, 3A4. 2C8 }\end{array}$ \\
\hline
\end{tabular}

* This effect was elicited by a daily dose of $40 \mathrm{mg}$ of lovastatin, pravastatin, simvastatin, atorvastatin, and fluvastatin, and by a daily dose of $0.3 \mathrm{mg}$ of cerivastatin in patients with hypercholesterolaemia.

Reproduced from Knopp, $N$ Engl F Med 1999;341:498-511, with permission of the publisher.

common than low HDL cholesterol or a combination of low HDL cholesterol and elevated triglycerides (with or without elevated LDL cholesterol). ${ }^{18}$ The recent Veterans Affairs high density lipoprotein cholesterol intervention trial (VA-HIT) study focused specifically on this population to evaluate gemfibrozil versus placebo in CHD patients with low HDL cholesterol, elevated triglycerides, and normal LDL cholesterol and total cholesterol concentrations. ${ }^{19}$ Gemfibrozil, a fibric acid derivative that raises HDL cholesterol and lowers triglycerides, with minimal effect on LDL cholesterol concentrations, was associated with significant reductions in recurrent coronary events. Some commentators have interpreted the VA-HIT results to indicate that, for this segment of the CHD population, statin treatment may not be the only or even the optimal choice for lipid regulating secondary prevention.

However, the clinical trial record indicates that statins also provide significant secondary preventive benefits in CHD patient populations with normal or mildly elevated total and LDL cholesterol concentrations. ${ }^{23}$ On this basis, many authorities recommend administration of statins to all patients with CHD, regardless of their specific lipid profile. At present, fibrates and niacin are the most likely adjunctive candidates for treating low HDL cholesterol and/or hypertriglyceridaemia, and niacin may also lower elevated concentrations of $\mathrm{Lp}(\mathrm{a})$ lipoprotein and small dense LDL. However, the gains in efficacy of such combination treatment must be weighed against potential deficits in safety, tolerability, and compliance. ${ }^{20}$

\section{PLEIOTROPIC EFFECTS}

Different statins appear to have favourable effects on a variety of non-lipid factors and processes that may influence both chronic disease progression and the onset of acute coronary events. ${ }^{21-23}$ Clinical studies have shown that all of the available statins help to restore impaired endothelial function and vasomotion, which are hallmarks of atherosclerosis. Experimental studies in animal models indicate that statin treatment stabilises atherosclerotic plaques by reducing lipid deposits and promoting the synthesis of structural proteins. All of the current statins, save pravastatin, have been shown to inhibit smooth muscle cell proliferation in vitro. There are two schools of thought on the value of this antiproliferative activity. On the one hand, smooth muscle cell proliferation and migration have been implicated as part of the atherogenic process. Conversely, such proliferation may help improve the structural integrity of plaques, making them less likely to rupture and thereby trigger a coronary event.

A lively debate has emerged between investigators who attribute these pleiotropic effects of statins to intrinsic ancillary properties of the drugs themselves, and those who contend that they are secondary phenomena consequent to LDL cholesterol reduction. A new long term mortality trial, bluntly but aptly entitled PROVE IT (pravastatin or atorvastatin evaluation and infection therapy), may clarify the situation. This study will compare maximum dosages of atorvastatin ( $80 \mathrm{mg} /$ day) versus pravastatin $(40 \mathrm{mg} / \mathrm{day})$. Atorvastatin is the most powerful LDL cholesterol reducing agent available today, whereas pravastatin has perhaps the most extensive literature associating it with various pleiotropic effects. The results are expected in about five years.

\section{IDEAL EFFICACY}

Major clinical trial data indicate that statins reduce initial or recurrent $\mathrm{CHD}$ events by $25-60 \%$, with an average reduction in overall mortality of about $30 \%$. The question has been raised that if these drugs are so effective, why are the clinical benefits experienced by only a minority of statin treated trial participants? The flaw in this logic is that no clinical trial can adequately reflect the multifaceted biology of real life, and to eliminate a disease that progresses over the course of a lifetime would require therapeutic intervention over a longer time frame than has been captured by any clinical trial. The efficacy of statins in secondary prevention compares favourably with that of other cardiovascular standards - for example, $\beta$ blockers and angiotensin converting enzyme (ACE) inhibitors. 
Table 2 Drugs and substances that interfere with the metabolism of statins

\begin{tabular}{|c|c|c|}
\hline Mechanism of action & Effect & Drug or substance \\
\hline $\begin{array}{l}\text { Inhibits cytochrome } \\
\text { P450 3A4 }\end{array}$ & $\begin{array}{l}\text { Raises serum drug } \\
\text { concentrations }\end{array}$ & $\begin{array}{l}\text { Clarithromycin, cyclosporine, tacrolimus, } \\
\text { delavirdine mesylate, ritonavir, } \\
\text { ketoconazole, fluoxetine, grapefruit juice, } \\
\text { miberfradil, nefazodone }\end{array}$ \\
\hline $\begin{array}{l}\text { Induces cytochrome } \\
\text { P450 3A4 }\end{array}$ & $\begin{array}{l}\text { Lowers serum drug } \\
\text { concentrations }\end{array}$ & $\begin{array}{l}\text { Barbiturates, carbamazepine, griseofulvin, } \\
\text { nafcillin, phenytoin, primidone, rifabutin, } \\
\text { rifampin, troglitazone }\end{array}$ \\
\hline $\begin{array}{l}\text { Inhibits cytochrome } \\
\text { P450 2C9 }\end{array}$ & $\begin{array}{l}\text { May raise serum fluvastatin } \\
\text { concentrations }\end{array}$ & $\begin{array}{l}\text { Amiodarone, cimetidine, fluoxetine, } \\
\text { fluvoxamine, isoniazid, itraconazole, } \\
\text { sulfinpyrazone, ticlopidine, zafirlukast }\end{array}$ \\
\hline $\begin{array}{l}\text { Induces cytochrome } \\
\text { P4502C9 }\end{array}$ & $\begin{array}{l}\text { May lower serum fluvastatin } \\
\text { concentrations }\end{array}$ & $\begin{array}{l}\text { Barbiturates, carbamazepine, phenytoin, } \\
\text { primidone, rifampin }\end{array}$ \\
\hline
\end{tabular}

Adapted from Knopp. ${ }^{16}$

Nevertheless, for patients with substantial hypercholesterolaemia, the lipid lowering potential of statins can be improved through more aggressive dosing in most patients, through combination treatment with other lipid regulating agents in selected patients, and, in general, through the development of more powerful and comprehensive "superstatin" drugs. For a new statin or other agent to qualify as superior, it should provide sufficient cholesterol lowering power to enable the broadest possible at-risk population to reach target cholesterol concentrations with an appropriate starting dose. It should be equally efficacious in all age, race, and sex groups, as well as in special risk populations such as patients with diabetes or hypertension. It should also reduce coronary events at least as well as those agents that have already been tested in clinical trials.

The ideal lipid regulating agent would offer primary benefits based on LDL cholesterol reduction, but would also have potent salutary effects on HDL cholesterol, triglycerides, and various emerging lipid subfractions and components, such as $\mathrm{Lp}$ (a) lipoprotein, small dense $\mathrm{LDL}, \mathrm{HDL}_{2}$ and $\mathrm{HDL}_{3}$, and cholesterol ester transfer protein. It would, in addition, combine safely and conveniently with other drugs designed to address the various risk factors that predispose to cardiovascular disease. Finally, the ideal agent would impact favourably on the physiology of the arterial wall and promote blood flow by improving endothelial function, reducing atherothrombosis, and modulating the inflammatory, immunologic, and proliferative factors contributing to disease progression and acute events. If most or even some of these efficacy criteria are met, we can project with confidence that long term reductions in CHD morbidity and mortality would exceed the current standards.

\section{Safety of statins}

Statins in general have an excellent safety record in both clinical trials and clinical practice. The most frequently reported adverse effects of statins involve gastrointestinal distress, muscle soreness, and disturbances of hepatic function. ${ }^{24}$ Frank hepatotoxicity and rhabdomyolysis are uncommon, even at high dosages (raised liver and enzyme valuesgreater than three times and 10 times the upper reference value, respectively-have been reported in less than $1 \%$ of treated subjects); however, they do occur more often with the statins that are metabolised via the cytochrome P450 enzyme system. ${ }^{16}$

Atorvastatin, cerivastatin, lovastatin, and simvastatin are metabolised primarily through the cytochrome P450 3A4 system. Fluvastatin is metabolised by the cytochrome P450 2C9 system. Pravastatin alone among the available statins does not involve cytochrome P450 enzymes in its metabolism. Some of the drugs and other substances (for example, grapefruit juice) that inhibit or induce cytochrome P450 enzymes and thus interfere with statin metabolism are presented in table 2 . In addition, warfarin concentrations may increase if it is given concurrently with fluvastatin.

Because of the hepatic focus of cholesterol lowering treatment, monitoring of serum aminotransferase concentrations is generally recommended for all patients receiving statins or other current lipid regulating drugs. Such monitoring is essential for patients receiving a statin along with a fibrate drug or niacin. These drugs greatly increase the risk of hepatic effects; therefore, high dosages of statins generally are not recommended in combination with fibrates or niacin. This caution may be particularly important in elderly patients, who tend to have increased susceptibility to hepatotoxicity.

From a safety standpoint, the ideal lipid regulating agent: would not be metabolised by the cytochrome P450 system; would not require monitoring or physician concern regarding potential hepatic complications; would be free of life threatening adverse effects, no matter how rare; and would be safe to administer at maximum dosages to all patients alone or in combination with other lipid regulating substances. It remains to be determined whether the superstatins will be associated with any adverse effects with long term use, although early indications suggest that concerns of this nature are unfounded.

\section{Appropriate use of statins}

Despite the overwhelming clinical trial evidence showing the benefits of statins, physician acceptance and patient compliance pose the greatest challenges to effective treatment. In clinical practice, these drugs remain dramatically underprescribed. ${ }^{25}{ }^{26}$ In fact, three late1990s analyses estimated that only about one third of the patents who might benefit from statin treatment in the USA, Europe, and Asia were actually receiving it. ${ }^{27-29}$ Even when statins are prescribed appropriately, patient compliance with treatment regimens is often unsatisfactory.$^{10}$ Failures of organisation, communication, physician perception, and patient education all contribute to a widespread loss of opportunities to prolong life and preserve health. ${ }^{26} 30$

The reluctance of physicians and patients to implement and adhere to evidence based treatment was demonstrated in the primary care setting by follow up analysis of the landmark WOSCOPS trial (J Shepherd, unpublished data) which indicated that less than two thirds of the patients who had suffered $\mathrm{CHD}$ events during the trial were still 
receiving statin treatment 2.5 years after its close. Actual practice diverged from well publicised guidelines and clinical trial evidence, even in this group of trial experienced physicians and patients.

Primary prevention presents serious challenges to physician acceptance and patient compliance. Initiation of chronic drug treatment in asymptomatic individuals can be a difficult barrier for both physician and patient to cross. Exaggerated news reports about the dangers of medical treatments or advances in nutritional therapy can further confuse the issues. Economics pose another major obstacle. Statin treatment of all individuals at moderately increased risk according to current guidelines would place an enormous burden on the health care system. This knowledge has a dampening effect that travels back down the line; the prevailing message is not to incur treatment costs in the absence of immediately identifiable need.

The intrinsic properties of a drug - even an ideal drug-cannot by themselves ensure appropriate utilisation. An ideal treatment may enhance physician and patient comfort by providing maximum efficacy and safety in the most convenient formulation. This would entail once daily administration of a single treatment that is not influenced by meals or time of day; that has minimal adverse effects and monitoring requirements; and that requires minimal dosage adjustments according to age, size, race, sex, or concomitant conditions. To make a real difference in utilisation, however, the ideal agent must be accompanied by a comprehensive programme of effective communication and educational tools.

\section{Conclusion}

Although statins today are the dominant class of lipid regulating drugs, they fall short of the ideal in several important respects (table 3 details the recommended attributes of the ideal lipid regulating agent).

Whether any of the new agents currently being developed can meet these high standards remains to be seen. It is hoped that investigational superstatins, such as AstraZeneca's ZD4522 and Nissan Chemical's NK-104, will surpass current statin capabilities in one or more of these categories. Other promising agents, such as microsomal triglyceride transfer protein inhibitors and cholesterol and bile acid absorption blockers, may ultimately complement advanced statins in a step towards ideal

Table 3 Attributes of the ideal lipid modifying agent

- Produce clinically significant and sustainable reductions in coronary events and overall mortality in both primary and secondary prevention in cost-effective fashion

- Bring majority of patients to recommended cholesterol concentrations with low starting dosage

- Produce significant elevations in HDL cholesterol and reductions in triglycerides

- Favourably affect the entire lipid profile

- Improve impaired endothelial function

- Broad ranging pleiotropic effects that mediate thrombotic inflammatory, immunologic, and proliferative disease mechanisms

- Safe and well tolerated at all dosages

- Once daily dosing, not dependent on meals or time of day combination treatment. The prospects for effective drug management of atherosclerosis have never been better. The outstanding question for the new millennium is, how will we pay for such treatment on a global basis?

1 Scandinavian Simvastatin Survival Study Group. Randomised trial of cholesterol lowering in 4444 patients with coronary heart disease: the Scandinavian simvastatin coronary heart disease: the Scandinavian
survival study (4S). Lancet 1994;344:1383-9.

2 Sacks FM, Moye LA, Davis BR, et al. Relationship between plasma LDL concentrations during treatment with pravastatin and recurrent coronary events in the cholesterol and recurrent events trial. Circulation 1998;97:1446-52.

3 The Long-Term Intervention with Pravastatin in Ischaemic Disease (LIPID) Study Group. Prevention of cardiovascular events and death with pravastatin in patients with coronary heart disease and a broad range of initial cholesterol levels. N Engl f Med 1998;339:1349-57.

4 Shepherd J, Cobbe SM, Ford I, et al, for the West of Scotland Coronary Prevention Study Group. Prevention of coronary heart disease with pravastatin in men with hypercholesterolemia. N Engl f Med 1995;333:1301-7.

5 Downs JR, Clearfield M, Weis S, et al, for the AFCAPS/ TexCAPS Research Group. Primary prevention of acute coronary events with lovastatin in men and women with average cholesterol levels. Results of AFCAPS/TexCAPS. fAMA 1998;279:1616-22.

6 The Post Coronary Artery Bypass Graft Trial Investigators. The effect of aggressive lowering of low-density lipoprotein cholesterol levels and low-dose anticoagulation on obstructive changes in saphenous-vein coronary-artery bypass tive changes in saphenous-vein coron
grafts. $N$ Engl $\mathcal{F}$ Med 1997;336:153-62.

7 Pitt B, Waters D, Brown WV, et al, for the Atorvastatin versus Revascularization Treatment Investigators. Aggressive lipid-lowering therapy compared with angioplasty in stable coronary artery disease. $N$ Engl f Med 1999;341:70-6.

8 World Health Organization. World health report. Geneva: WHO, 1998.

9 Expert Panel on Detection, Evaluation and Treatment of High Blood Cholesterol in Adults: National Cholesterol Education Program. Summary of the second report of the expert panel on detection, evaluation and treatment of high expert panel on detection, evaluation and treatment of high
blood cholesterol in adults (adult treatment panel II). fAMA 1993;269:3015-23.

10 Second Joint Task Force of European and Other Societies on Coronary Prevention. Prevention of coronary heart disease in clinical practice. Recommendations of the second joint task force of European and other societies on coronary prevention. Eur Heart $\mathcal{F}$ 1998;19:1434-503.

11 Martin MJ, Hulley SB, Browner WS, et al. Serum cholesterol, blood pressure, and mortality: implications from a cohort of 361,622 men. Lancet 1986;ii:933-6.

12 Sacks FM, Pfeffer MA, Moye LA, et al, for the Cholesterol and Recurrent Events Trial Investigators. The effect of pravastatin on coronary events after myocardial infarction
in patients with average cholesterol levels. $N$ Engl $\mathcal{F ~ M e d ~}$ 1996;335:1001-9.

13 West of Scotland Coronary Prevention Study Group. Influence of pravastatin and plasma lipids on clinical events in the West of Scotland coronary and prevention study (WOSCOPS). Circulation 1998;97:1440-5

14 Gotto AM Jr, Whitney E, Stein EA, et al. Relation between baseline and on-treatment lipid parameters and first acute major coronary events in the Air Force/Texas coronary atherosclerosis prevention study (AFCAPS/TexCAPS). Circulation 2000;101:477-84.

15 Pedersen TR. Aggressive lipid-lowering therapy: a clinical imperative. Eur Heart f 1998;19(suppl M):M15-21.

16 Knopp RH. Drug treatment of lipid disorders. N Engl f Med 1999;341:498-511.

17 Wierzbicki AS, Lumb PJ, Chik G, et al. Comparison of therapy with simvastatin $80 \mathrm{mg}$ and atorvastatin $80 \mathrm{mg}$ in patients with familial hypercholesterolemia. Int $\mathcal{F}$ Clin Pract 1999;53:609-11.

18 Genest J Jr, McNamara JR, Ordovas JM, et al. Lipoprotein cholesterol, apolipoprotein A-I and B and lipoprotein (a) abnormalities in men with premature coronary artery disease. F Am Coll Cardiol 1992;19:792-802.

19 Rubins HR, Robins SJ, Collins D, et al, for the Veterans Affairs High-Density Lipoprotein Cholesterol Intervention Trial Study Group. Gemfibrozil for the secondary prevention of coronary heart disease in men with low levels of tion of coronary heart disease in men with low levels of 1999;341:410-18.

20 Schectman G, Hiatt J. Drug therapy for hypercholesterolemia in patients with cardiovascular disease: factors limiting achievement of lipid goals. Am F Med 1996;100:197204.

21 Rosenson RS, Tangney CC. Antiatherothrombotic properties of statins: implications for cardiovascular event reduction. $\mathcal{F} A M A$ 1998;279:1643-50.

22 Vaughan CJ, Murphy MB, Buckley BM. Statins do more than just lower cholesterol. Lancet 1996;348:1079-82.

23 Wheeler DC. Are there potential non-lipid-lowering uses of statins? Drugs 1998;56:517-22.

24 Anon. Physician's desk reference. Montvale, New Jersey: Medical Economics, 1999. 
25 EUROASPIRE Study Group. A European Society of Cardiology survey of secondary prevention of coronary heart disease: principal results. Eur Heart f 1997;18:156982.

26 Shepherd J, Alcalde V, Belfort P-A, for the HELP Study Group. International comparison of awareness and attitudes towards coronary risk factor reduction: the HELP study. F Cardiovasc Risk 1997;4:373-84.

27 American Heart Association. 1998 Heart and stroke statistical update. Dallas, Texas: AHA; 1998.
28 EUROASPIRE Study Group. EUROASPIRE. A European Society of Cardiology survey of secondary prevention of eart disease: principal results. Eur Heart f7 1997;18:1569 82

29 Keech A. Risk factor management in CHD patients in Asia: current status. Atherosclerosis 1998;136(suppl):31

30 Shea S, Gemson DH, Mossel P. Management of high blood cholesterol by primary care physicians: diffusion of the national cholesterol education program adult treatment panel guidelines. F Gen Intern Med 1990;5:327-34.

\section{IMAGES IN CARDIOLOGY}

\section{Ostial left anterior descending artery stenosis undetected on angiography: role of intravascular ultrasound}

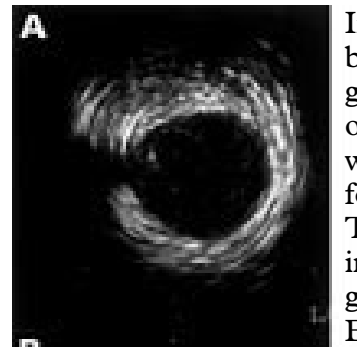

Intravascular ultrasound can be used to assess borderline lesions seen on coronary angiography. A 50 year old woman with angina and objective evidence of ischaemia in the anterior wall underwent multiple coronary angiograms following recurrent admissions with angina. These failed to demonstrate any specific lesion in the left anterior descending artery that might guide an intravascular ultrasound examination.

B

Examination of the left anterior descending
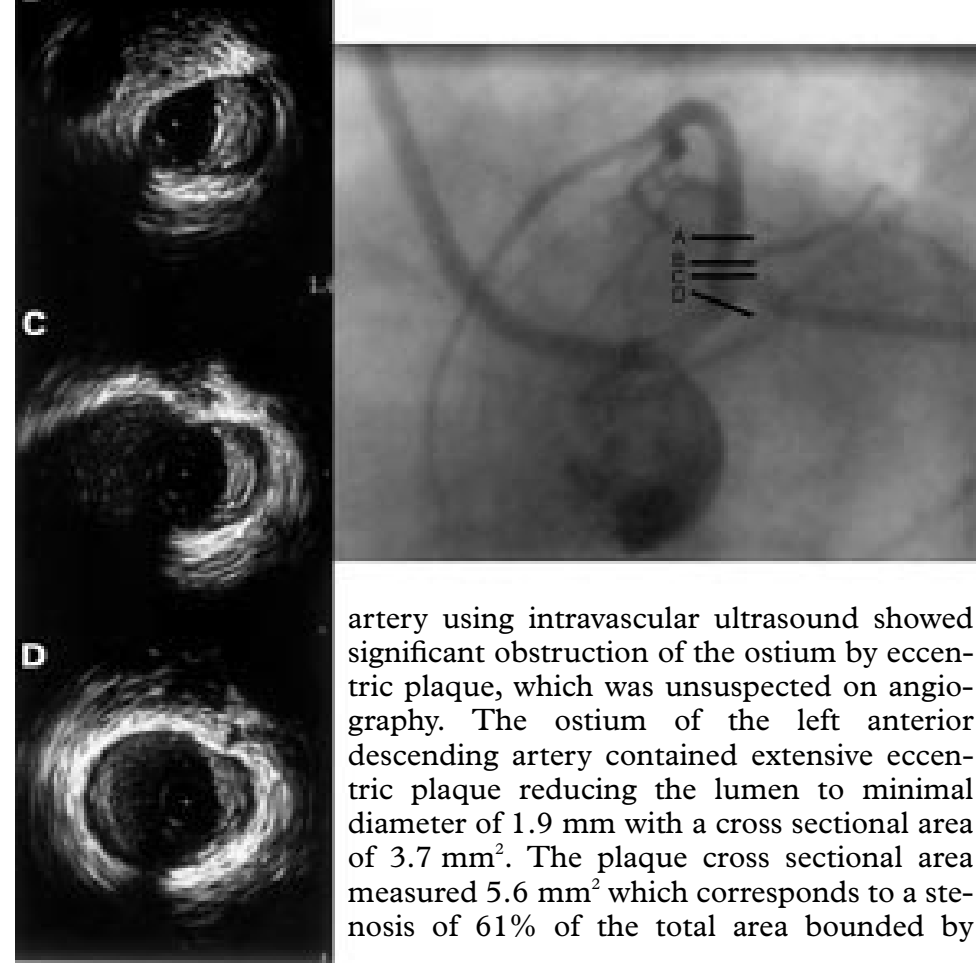

artery using intravascular ultrasound showed significant obstruction of the ostium by eccentric plaque, which was unsuspected on angiography. The ostium of the left anterior descending artery contained extensive eccentric plaque reducing the lumen to minimal diameter of $1.9 \mathrm{~mm}$ with a cross sectional area of $3.7 \mathrm{~mm}^{2}$. The plaque cross sectional area measured $5.6 \mathrm{~mm}^{2}$ which corresponds to a stenosis of $61 \%$ of the total area bounded by the media. The maximal diameter of the ostium measured $4 \mathrm{~mm}$ and the percentage stenosis was $53 \%$. The plaque extended proximally into the left main coronary artery but did not significantly obstruct the lumen. The left anterior descending artery ostial lesion was fairly short in length and the remainder of the proximal, mid and distal segments of the left anterior descending artery appeared free of significant atheroma.

Pathological studies show that atherosclerotic lesions have a distinct spatial distribution around coronary bifurcation. In the case of the left main bifurcation, lesions were frequently found on the outer walls of the proximal branches particularly the left anterior descending artery, where wall shear stress was low. The flow divider and inner walls of the arterial branches were found to be relatively free of atherosclerosis. In fact this was exactly the pattern of distribution of atherosclerosis in our patient with eccentric plaque limited to the outer sector of the left anterior descending artery opposite the branching point of the circumflex artery.

Coronary angiography has limitations in assessing the three dimensional spatial arrangement of coronary bifurcations and hence short ostial lesions at these sites may be missed on angiography. Intravascular ultrasound is the technique of choice for detecting such lesions in patients with recurrent angina and objective evidence of ischaemia, in whom angiography may not reveal any obstructive lesion.

$\mathrm{T}$ W KOH A MONEY-KYRLE T CRAKE 